International Journal of Pure and Applied Mathematics

Volume 92 No. 2 2014, 297-303

ISSN: 1311-8080 (printed version); ISSN: 1314-3395 (on-line version)

url: http://www.ijpam.eu

doi: http://dx.doi.org/10.12732/ijpam.v92i2.11

ijpam.eu

\title{
SECOND NOTE ON THE SHAPE OF $S$-CONVEXITY
}

\author{
I.M.R. Pinheiro \\ P.O. Box 12396 A'Beckett St. \\ Melbourne, Victoria, 8006, AUSTRALIA
}

Abstract: This note supplements First Note on the Shape of $S$-convexity. We here deal with the negative pieces of the real functions.

AMS Subject Classification: 26A51

Key Words: analysis, convexity, definition, $S$-convexity, geometry, shape

\section{Introduction}

We seem to have progressed quite a lot with the wording of the analytical definitions for the $S$-convex real functions (see, for instance, [1], paper that comes straight after [2] in our series on improvements of the wording of the definition of the $S$-convexity phenomenon). One of the resulting analytical definitions in [1] is:

Definition 1. A function $f: X->\Re$, where $|f(x)|=-f(x)$, is told to belong to $K_{s}^{2}$ if the inequality

$$
f(\lambda x+(1-\lambda)(x+\delta)) \leq \lambda^{\frac{1}{s}} f(x)+(1-\lambda)^{\frac{1}{s}} f(x+\delta)
$$

holds $\forall \lambda / \lambda \in[0,1] ; \forall x / x \in X ; s=s_{2} / 0<s_{2} \leq 1 ; X / X \subseteq \Re_{+} \wedge X=[a, b]$; $\forall \delta / 0<\delta \leq(b-x)$. 
Remark 1. If the inequality is obeyed in the supplementary ${ }^{1}$ situation by $f$, then $f$ is said to be $s_{2}$-concave.

We now need to have a geometric definition for this group of $S$-convex functions.

The geometric definition of the convex functions has been worded by us in [1] in the following way:

Definition 2. A real function $f: X->Y$ is called convex if and only if, for all choices $\left(x_{1} ; y_{1}\right)$ and $\left(x_{2} ; y_{2}\right)$, where $\left\{x_{1}, x_{2}\right\} \subset X,\left\{y_{1}, y_{2}\right\} \subset Y$, $Y=I m_{f}$, and $x_{1} \neq x_{2}$, it happens that the chord drawn between $\left(x_{1} ; y_{1}\right)$ and $\left(x_{2} ; y_{2}\right)$ does not contain any point with height, measured against the vertical Cartesian axis, that be inferior to the height of its horizontal equivalent in the curve representing the ordered pairs of $f$ in the interval considered for the chord in terms of distance from the origin of the Cartesian axis.

We would like to word the geometric definition of the $S$-convex functions in a similar way:

Definition 3. A real function $f: X->Y$, for which $|f(x)|=-f(x)$, is called $S$-convex if and only if, for all choices $\left(x_{1} ; y_{1}\right)$ and $\left(x_{2} ; y_{2}\right)$, where $\left\{x_{1}, x_{2}\right\} \subset X,\left\{y_{1}, y_{2}\right\} \subset Y, Y=I m_{f}$, and $x_{1} \neq x_{2}$, it happens that the line drawn between $\left(x_{1} ; y_{1}\right)$ and $\left(x_{2} ; y_{2}\right)$ by means of the expression $(1-\lambda)^{\frac{1}{s}} y_{1}+\lambda^{\frac{1}{s}} y_{2}$, where $\lambda \in[0,1]$, does not contain any point with height, measured against the vertical Cartesian axis, that be inferior to the height of its horizontal equivalent in the curve representing the ordered pairs of $f$ in the interval considered for the line in terms of distance from the origin of the Cartesian axis.

To prove that the geometric rule for pertinence to the $S$-convex class of functions is the one that we here present, we remind the reader that $(1-\lambda)^{\frac{1}{s}} y_{1}+$ $\lambda^{\frac{1}{s}} y_{2} \geq(1-\lambda) y_{1}+\lambda y_{2}$ due to the allowed (by the definition) values for $\lambda, s$, $y_{1}$, and $y_{2}$.

We prove that the geometric limiting line for $S$-convexity is continuous in Section 2.

In Section 3, we prove that, as $s$ decreases in value (as the distance from the convexity limiting line is increased), the length of the limiting line increases, therefore we prove that we have more functions in the 1/4-convex class than in the 1/2-convex class, for instance, what provides us with certainty that $S$-convexity is a proper extension of convexity, also geometrically speaking.

\footnotetext{
${ }^{1}$ Supplementary here means ' $>$ ', not ' $\geq$ '.
} 


\section{Continuity}

We now prove that the function $f(\lambda)=(1-\lambda)^{\frac{1}{s}} y_{1}+\lambda^{\frac{1}{s}} y_{2}$ is continuous through a few theorems from Real Analysis.

We know, for instance, that both the sum and the product of two continuous functions are continuous functions (see, for instance, [3]). Notice that $\lambda^{\frac{1}{s}}$ is continuous, given that $0 \leq \lambda \leq 1$ and $0<s \leq 1$. $y_{1}$ and $y_{2}$ are constants, therefore could be seen as constant functions, which are continuous functions. $(1-\lambda)^{\frac{1}{s}}$ is continuous due to the allowed values for $\lambda$ and $s$. As a consequence, $(1-\lambda)^{\frac{1}{s}} y_{1}+\lambda^{\frac{1}{s}} y_{2}$ is continuous.

Notice that $f(\lambda)=(1-\lambda)^{\frac{1}{s}} y_{1}+\lambda^{\frac{1}{s}} y_{2}$ is $C^{\infty}$, that is, is smooth (see [4], for instance).

We do notice that we will have problems, for instance, with the first derivative of $f(\lambda)$ of the sort $n^{\infty}$ (see [5], for instance) or $0^{0}$, when $\lambda \in\{0,1\}$ and $s=0$ or $s->\infty$, but, in excluding 0 from our set of possible values for $s$, and limiting it adequately, what is done in the definition of $S$-convex functions, we disappear with those problems.

We say that $s \in(0,1)$ when $f^{\prime}(\lambda)=-\frac{1}{s}(1-\lambda)^{\frac{1}{s}-1} y_{1}+\frac{1}{s} \lambda^{\frac{1}{s}-1} y_{2}$ and $s=1$ when $f^{\prime}(\lambda)=y_{2}-y_{1}$.

Because the coefficients that form the convexity limiting line use $100 \%$ split between the addends and form straight lines and the coefficients that form the $S$-convexity limiting line use more than $100 \%$ or $100 \%$ split between the addends, given that $-(1-\lambda)^{\frac{1}{s}} \geq-(1-\lambda)$ and $-\lambda^{\frac{1}{s}} \geq-\lambda$ (we are using the negativity of the function here), we know that the limiting line for $S$-convexity lies always above or over the limiting line for convexity, and contains two points that always belong to both the convexity and the $s$-convexity limiting lines (first and last or $\left(x_{1} ; y_{1}\right)$ and $\left.\left(x_{2} ; y_{2}\right)\right)$.

We now have then proved, in a definite manner, also in the shape of a paper, that our limiting line for the $S$-convexity phenomenon is smooth, continuous, and located above or over the limiting line for the convexity phenomenon. Our $S$-convexity limiting line should also be concave when seen from the limiting convexity line for the same points $\left(\left(x_{1} ; y_{1}\right)\right.$ and $\left.\left(x_{2} ; y_{2}\right)\right)$ (taking away the cases in which $y_{1}=y_{2}=0$ or $\left.s=1\right)$, as we asserted to be the case in our talks at the Victoria University of Technology (2001) and at the Adelaide University (2005). 


\section{Arc Length}

Arc length is defined as the length along a curve,

$$
s \equiv \int_{\gamma}|d l|,
$$

where $\mathrm{dl}$ is a differential displacement vector along a curve $\gamma$ (see [6]).

In Cartesian coordinates, that means that the Arc Length of a curve is given by

$$
p \equiv \int_{a}^{b} \sqrt{1+f^{\prime} 2(x)} d x
$$

whenever the curve is written in the shape $r(x)=x \hat{\boldsymbol{x}}+f(x) \hat{\boldsymbol{y}}$.

Our limiting curve for $S$-convexity could be expressed as a function of $\lambda$ in the following way:

$$
f(\lambda)=(1-\lambda)^{\frac{1}{s}} y_{1}+\lambda^{\frac{1}{s}} y_{2} .
$$

In deriving the above function in terms of $\lambda$, we get:

$$
f^{\prime}(\lambda)=-\frac{1}{s}(1-\lambda)^{\frac{1-s}{s}} y_{1}+\frac{1}{s} \lambda^{\frac{1-s}{s}} y_{2} .
$$

With this, our arc length formula will return:

$$
p \equiv \int_{0}^{1} \sqrt{1+\left[-\frac{1}{s}(1-\lambda)^{\frac{1-s}{s}} y_{1}+\frac{1}{s} \lambda^{\frac{1-s}{s}} y_{2}\right]^{2}} d \lambda .
$$

We will make use of a constant function, and we know that every constant function is convex, therefore also $S$-convex (for every allowed value of $s$ ), to study the limiting line for $S$-convexity better.

We choose $f(x)=-1$ to work with (this function is suitable because $|f(x)|=|-1|=1=-f(x))$.

We then have:

$$
p \equiv \int_{0}^{1} \sqrt{1+\left[\frac{1}{s}(1-\lambda)^{\frac{1-s}{s}}-\frac{1}{s} \lambda^{\frac{1-s}{s}}\right]^{2}} d \lambda .
$$

Notice that $s \rightarrow 0 \Rightarrow p \rightarrow$ indeterminate and $s=1 \Rightarrow p=1$.

Notice that 0.25 will become 0.0625 when raised to $\frac{1}{0.5}$ and its supplement through the formula $(1-\lambda), 0.75$, will become 0.5625 .

In convexity, our results would have been 0.25 and 0.75 instead, that is, $75 \%$ and $25 \%$ less in negativity is gotten with $S$-convexity, respectively. 
We finish this section with a table ${ }^{2}$ containing three of the possible values for $s$ and their respective arc lengths (good approximations. Only the first value is precise) for the situation in which $f(x)$ is replaced with -1 in the arc length formula:

\begin{tabular}{cc}
\hline \hline$s$ & Arc Length \\
\hline 1 & 1 \\
0.5 & 1.57 \\
0.25 & 2 \\
\hline \hline
\end{tabular}

We notice that the naive formula from [7] gives us an excellent approximation to our arc length for the $s$-convexity limiting line, so that there is a good chance that we can use the naive formula to calculate the arc length in this situation always.

\section{Maximum Height}

The maximum height of the $s$-convexity limiting curve is reached when $\lambda=0.5$ if $f$ is constant and $|f(x)|=-f(x)$ because the first derivative of the function describing the limiting line gives us zero for $\lambda=0.5$ and changes sign from positive to negative there.

\section{Conclusion}

This paper and [8], together, provide us with a geometric definition for the phenomenon $S$-convexity when it happens inside of the Universe of the Real Numbers.

We here supplement [8] by dealing with the cases in which the function is negative, present alternative argumentation as to why the shape of $S$-convexity is what we have declared it to be in our 2001 talk at the Victoria University of Technology, talk given to the members of the Research Group on Inequalities and Applications that there were working on a regular basis, and in our 2005 talk at the University of Adelaide, talk given to an academic audience working,

\footnotetext{
${ }^{2}$ The first value for Arc Length in the table has been attained through simple substitution in the formula. The second value has been attained through using the formula for circumference length. The third value has been attained through the naive formula from [7]. Hand measurement has returned 2 as a result for the third value as well.
} 
or interested in working, with Geometry, and present a conjecture of major importance that connects the $s$ in $S$-convexity with the arc length function and gives us a relationship that determines how bent the limiting curve is in function of the value of $s$ (the conjecture is formed from the analysis of a few experimental results).

Because of our calculations, and also because of a few graphical simulations, we get to the conclusion that the definition of the phenomenon $S$-convexity, in what regards negative real functions, is not yet perfect, since the exponent referring to the percentages taken from the main heights, let's say, is not the best (for instance, we are getting a bit more rope here than what we got in the first case). Our definition for the negative case seems to be a very good approximation, however, like it seems to be the closest thing that we can get with nice exponents.

Future work may present alternative exponents.

\section{References}

[1] M.R. Pinheiro, Minima domain intervals and the $S$-convexity, as well as the convexity, phenomenon, Advances in Pure Mathematics, 3 (2013).

[2] M.R. Pinheiro, First note on the definition of $S_{2}$-convexity, Advances in Pure Mathematics, 1 (2011), 1-2.

[3] L.S. Hush, Continuous Functions, Retrieved December 8, 2011 from http://archives.math.utk.edu/visual.calculus/1/continuous.5/index.html (1995).

[4] Planetmath authors, Continuously Differentiable, Retrieved December 9, 2011 from http://planetmath.org/encyclopedia/ ContinuouslyDifferentiable.html (2011).

[5] E.W. Weisstein, Indeterminate, Retrieved December 12, 2010 from http://mathworld.wolfram.com/Indeterminate.html (2013).

[6] E.W. Weisstein, Arc Length, Retrieved December 5, 2010 from http://mathworld.wolfram.com/ArcLength.html (2002).

[7] G.P. Michon, Perimeter of an Ellipse, Retrieved December 12, 2011 from http://www.numericana.com/answer/ellipse.htm (2000). 
[8] M.R. Pinheiro, First note on the shape of S-convexity, International Journal of Pure and Applied Mathematics, 90 (2014), 101-107, doi: 10.12732/ijpam.v90i1.12. 
